\title{
Canonical partition function for the hydrogen atom via the Coulomb propagator
}

\author{
S. M. Blinder \\ Department of Chemistry, University of Michigan, Ann Arbor, Michigan 48109-1055
}

(Received 7 October 1994; accepted for publication 2 December 1994)

The electronic partition function for the hydrogen atom is derived by integration over the recently-available Coulomb propagator. This provides a resolution to an old paradox in statistical mechanics: the apparent divergence of the hydrogen partition function. Electronic excitation does not contribute significantly to the standard-state partition function until temperatures of the order of $5000 \mathrm{~K}$. Thereafter, the continuum, with its immense density of states, makes the dominant contribution. From the discrete and continuum contributions to the partition function, a modification of the Saha-Boltzmann equation for the ionization equilibrium in atomic hydrogen is derived. (C) 1995 American Institute of Physics.

\section{INTRODUCTION}

A long-troublesome paradox in statistical thermodynamics concerns the electronic contribution to the canonical-ensemble partition function for a hydrogen atom. This has been the subject of an extensive literature, including contributions by some giants of Twentieth Century science. ${ }^{1} \mathrm{By}$ a standard formula of statistical mechanics,

$$
q=\sum_{n} g_{n} e^{-\beta E_{n}}, \quad \beta \equiv 1 / k T
$$

If one naively puts in the hydrogenic energies $E_{n}=-Z^{2} E_{\mathrm{H}} / 2 n^{2}\left(E_{\mathrm{H}}=m e^{4} / \hbar^{2}=1\right.$ hartree $)$ and degeneracies (apart from spin) $g_{n}=n^{2}$ the electronic partition function is given by

$$
q_{\mathrm{elec}}=e^{-\beta Z^{2} E_{\mathrm{H}} / 2} \sum_{n=1}^{\infty} n^{2} e^{\beta Z^{2} E_{\mathrm{H}} / 2 n^{2}}=\infty,
$$

a divergent sum, even without taking account of continuum states. The factor $e^{-\beta Z^{2} E_{\mathrm{H}} / 2}$ defines the energy zero as the $n=1$ electronic ground state. Equation (2) notwithstanding, $q_{\text {elec }} \approx 1$ for all practical purposes under terrestrial conditions.

The source of the above paradox is fairly obvious. The molecular partition function depends on the temperature and volume. But the wave functions of the hydrogen atom extend, in principle, to infinity, overrunning any finite volume. Equation (2) comes from integrating every $\left|\psi_{n}\right|^{2}$ over all space, hence implying an infinite volume. Gibbs ${ }^{2}$ warned against this difficulty for cases "in which the system ... can be distributed in unlimited space" singling out, in fact, the attractive inverse-square law of force.

We shall adopt as a defining ansatz of the canonical cnscmble, very likely in accord with Gibbs' original intention, that each member of the ensemble be enclosed by a nominal boundary not necessarily representing a physical barrier. This is, in fact, more explicit in the case of the grand canonical ensemble, in which molecules can freely enter or leave through the defined boundary of the system. The partition function is accordingly defined as the volume integral over the diagonal elements of the quantum-mechanical density matrix, viz., 


$$
q(T, V)=\int_{V} K(\mathbf{r}, \mathbf{r}, \beta) d^{3} \mathbf{r}
$$

where

$$
K\left(\mathbf{r}_{1}, \mathbf{r}_{2}, \boldsymbol{\beta}\right)=\sum_{n} \psi_{n}\left(\mathbf{r}_{1}\right) \psi_{n}^{*}\left(\mathbf{r}_{2}\right) e^{-\beta E_{n}}
$$

This has the same form as the quantum-mechanical propagator ${ }^{3}$ with $\beta$ in place of $i t / \hbar$. We should emphasize that the complete set of eigenfunctions $\psi_{n}(\mathbf{r})$ pertain to the system in infinite space, notwithstanding the volume cutoff in Eq. (4).

The partition function for a free particle works out easily from Eq. (3). The propagator is

$$
K_{0}\left(\mathbf{r}_{1}, \mathbf{r}_{2}, \beta\right)=\left(m / 2 \pi \beta \hbar^{2}\right)^{3 / 2} e^{-m\left(\mathbf{r}_{1}-\mathbf{r}_{2}\right)^{2} / 2 \hbar \beta} .
$$

Setting $\mathbf{r}_{2}=\mathbf{r}_{1}$ and integrating over volume then gives the well-known translational partition function for a perfect gas:

$$
q_{0}(T, V)=\left(m / 2 \pi \beta \hbar^{2}\right)^{3 / 2} V=\left(2 \pi m k T / h^{2}\right)^{3 / 2} V .
$$

Note that the eigenvalue spectrum remains continuous since no physical boundary is imposed on the system. The conventional derivation, leading to the same result, sums over discrete particlein-a box eigenstates, which do vanish at the boundary. The volume $V$ in this case represents an actual container, which, as it becomes very large, leads to a quasicontinuum.

For the hydrogen atom, taking explicit account of discrete and continuum contributions:

$$
K(\mathbf{r}, \mathbf{r}, \beta)=\sum_{n, l, m}\left|\psi_{n, l, m}(\mathbf{r})\right|^{2} e^{\beta Z^{2} E_{\mathrm{H}} / 2 n^{2}}+\sum_{l, m} \int_{0}^{\infty}\left|\psi_{k, l, m}(\mathbf{r})\right|^{2} e^{-\beta \hbar^{2} k^{2} / 2 m} d k
$$

An analytic representation for the Coulomb propagator was derived by the author a couple of years ago, ${ }^{4}$ so that the partition function can now be evaluated, in principle, by integration over a volume $V$.

Previous approaches to the Coulomb partition-function paradox ${ }^{1}$ have typically involved its modification or redefinition, for example, by assuming a finite atomic volume or by truncating the infinite sum. Alternatively, a shielded Coulomb potential or many-particle effects have been introduced. The so-called Planck-Larkin partition function for a hydrogenic plasma ${ }^{5}$

$$
q=\sum_{n=1}^{\infty} n^{2}\left(e^{\left.-\beta E_{n}-1+\beta E_{n}\right)}\right.
$$

is convergent, but evidently undercounts the number of contributing states. ${ }^{6}$ In our approach to the resolution of this paradox, we preserve the original definition of the canonical partition function and explicitly evaluate the sum over states by making use of the Coulomb propagator.

In the remainder of this paper we shall employ atomic units $\hbar=m=|e|=1$ and assume infinite nuclear mass. Lengths are expressed in bohrs, $a_{0}=\hbar^{2} / m e^{2}$, and energies in hartrees, $E_{\mathrm{H}}=m e^{4} / \hbar^{2}=e^{2} / a_{0}$. We also redefine $\beta$ as a dimensionless parameter, whereby

$$
\beta \equiv E_{\mathrm{H}} / k T=315774 / T
$$

with $T$ in Kelvins. 


\section{ONE-DIMENSIONAL HYDROGEN ATOM}

Before considering the hydrogen atom itself, a preliminary étude is instructive. A onedimensional attractive deltafunction potential $V(x)=-Z \delta(x)$ is somewhat analogous to a Coulomb potential. It allows but one bound state $\psi_{0}(x)=Z^{1 / 2} e^{-Z|x|}$ with energy $E_{0}=-Z^{2} / 2$, reminiscent of a hydrogenic $1 s$ orbital. All other solutions are continuum eigenstates with $E \geqslant 0$. We have derived the following propagator for the "deltahydrogen" atom: ${ }^{7}$

$$
\begin{gathered}
K\left(x, x^{\prime}, \beta\right)=K_{0}\left(x, x^{\prime}, \beta\right)+\frac{Z}{2} e^{-X^{2} / 2 \beta} e^{u^{2}} \text { erfc } u, \\
X \equiv|x|+\left|x^{\prime}\right|, \quad u \equiv(X-Z \beta) / \sqrt{2 \beta},
\end{gathered}
$$

where $K_{0}$ is the one-dimensional free-particle propagator

$$
K_{0}\left(x, x^{\prime}, \beta\right)=(2 \pi \beta)^{-1 / 2} e^{-\left(x-x^{\prime}\right)^{2} / 2 \beta},
$$

and erfc, the complementary error function

$$
\operatorname{erfc} u \equiv 1-\operatorname{erf} u=\frac{2}{\sqrt{\pi}} \int_{u}^{\infty} e^{-z^{2}} d z
$$

The partition function is readily derived from Eq. (10) by integration over a length $L$ to give

$$
q=(2 \pi \beta)^{-1 / 2} L+\frac{1}{2}\left[e^{\beta Z^{2} / 2} \operatorname{erfc}\left(-\sqrt{\frac{\beta}{2}} z\right)-1\right]
$$

apart from terms which vanish as $L \rightarrow \infty$. The bound-state contribution to the partition function, equal to a single term $e^{\beta Z^{2} / 2}$, is partially cancelled by the continuum contribution. At the same time, the continuum splits out a free-particle function. As we shall see, an analogous thing happens in the hydrogen-atom partition function.

\section{COULOMB PARTITION FUNCTION}

To make this paper self-contained, we shall refer to parts of our derivation of the Coulomb propagator. ${ }^{4}$ See Ref. 4 for more complete details.

The Green's function for a quantum-mechanical system is defined by the following spectral representation in terms of the complete set of eigenstates:

$$
G^{+}\left(\mathbf{r}_{1}, \mathbf{r}_{2}, E\right)=\sum_{n} \frac{\psi_{n}\left(\mathbf{r}_{1}\right) \psi_{n}^{*}\left(\mathbf{r}_{2}\right)}{E-E_{n}+i \epsilon}
$$

the sum being understood to run over both discrete and continuum states, as appropriate. The superscript "+" refers to the retarded Green's function and is obtained with an infinitessimal positive imaginary $i \epsilon$ added to the parameter $E$. The nonrelativistic Coulomb Green's function was first derived in closed form by Hostler $^{8}$ in 1963. It can be expressed as follows: ${ }^{9}$

$$
G^{+}\left(\mathbf{r}_{1}, \mathbf{r}_{2}, E\right)=G^{+}(x, y, k)=-\frac{1}{\pi(x-y)}\left(\frac{\partial}{\partial x}-\frac{\partial}{\partial y}\right) g^{+}(x, y, k),
$$

where 


$$
g^{+}(x, y, k)=(i k)^{-1} \Gamma(1-i \nu) M_{i \nu}(-i k y) W_{i \nu}(-i k x)
$$

the latter function representing a pseudo-one-dimensional Coulomb system. The coordinate variables $x$ and $y$ are defined by

$$
x \equiv r_{1}+r_{2}+r_{12}, \quad y \equiv r_{1}+r_{2}-r_{12} .
$$

The energy is related to the wave number $k$ by

$$
E=\hbar^{2} k^{2} / 2 m=k^{2} / 2 \text {. }
$$

Also,

$$
\nu \equiv Z / k
$$

$M$ and $W$ are Whittaker functions as defined by Buchholz. ${ }^{10}$ For brevity, we write $M_{i \nu}$ and $W_{i \nu}$ in place of $M_{i \nu, 1 / 2}$ and $W_{i \nu, 1 / 2}$, respectively.

The corresponding Coulomb propagator has a spectral representation such as Eq. (4) and is thus a Fourier transform of the Green's function. Specifically, ${ }^{11}$

$$
K=\frac{i}{2 \pi} \int_{-\infty}^{\infty}\left(G^{+}-G^{-}\right) e^{-i E t} d E
$$

Assuming a structure for $K$ analogous to (15),

$$
K(x, y, t)=-\frac{1}{\pi(x-y)}\left(\frac{\partial}{\partial x}-\frac{\partial}{\partial y}\right) k(x, y, t)
$$

we have

$$
k=\frac{i}{2 \pi} \int_{-\infty}^{\infty}\left(g^{+}-g^{-}\right) e^{-i E t} d E
$$

Carrying out the integration in the complex plane, the result is

$$
k(x, y, \beta)=\sum_{n=1}^{\infty} Z_{n} M_{n}\left(Z_{n} x\right) M_{n}\left(Z_{n} y\right) e^{\beta Z_{n}^{2} / 2}+\frac{i}{2 \pi} \int_{-\infty}^{\infty} g^{+}(x, y, k) e^{-k^{2} \beta / 2} k d k,
$$

with two parts corresponding to the discrete spectrum and the continuum. We have introduced the abbreviation $Z_{n} \equiv Z / n$.

To carry out the integration in Eq. (23), we represent $g^{+}(x, y, k)$ in a Mittag-Leffler or rational fractional expansion ${ }^{12}$ as follows:

$$
\begin{aligned}
g^{+}(x, y, k) & =-i \sum_{n=1}^{\infty}\left(k-i Z_{n}\right)^{-1} M_{n}(-i k x) M_{n}(-i k y) \\
& =g_{0}^{+}(x, y, k)+\sum_{n=1}^{\infty} \frac{Z_{n} M_{n}(-i k x) M_{n}(-i k y)}{k\left(k-i Z_{n}\right)}
\end{aligned}
$$


This is also known as the Sturmian expansion of the Green's function ${ }^{13}$ since it contains hydrogenic functions with arguments independent of quantum number $n$, in contrast to Coulomb eigenfunctions, which involve $n$ in both index and argument. For $Z=0$, Eq. (24) reduces to Watson's formula $^{14}$ for the free-particle Green's function

$$
g_{0}^{+}(x, y, k)=(i k)^{-1} M_{0}(-i k y) W_{0}(-i k x)=(i k)^{-1} \sum_{n=1}^{\infty} M_{n}(-i k x) M_{n}(-i k y)
$$

Substituting (24) in (23) results in

$$
\begin{aligned}
k(x, y, \beta)= & \sum_{n=1}^{\infty} Z_{n} M_{n}\left(Z_{n} x\right) M_{n}\left(Z_{n} y\right) e^{\beta Z_{n}^{2} / 2}+k_{0}(x, y, \beta) \\
& +\frac{i}{2 \pi} \sum_{n=1}^{\infty} \int_{-\infty}^{\infty} \frac{Z_{n} M_{n}(-i k x) M_{n}(-i k y)}{k-i Z_{n}} e^{-\beta k^{2} / 2} d k .
\end{aligned}
$$

At this point, we transform to the three-dimensional propagator, using Eq. (21). Specifically, we need the diagonal elements of the propagator, with $\mathbf{r}_{1}=\mathbf{r}_{2}=\mathbf{r}$, corresponding to $x=y=2 r$. This involves the following limit:

$$
\begin{aligned}
& \lim _{x, y \rightarrow 2 r}\left\{-\frac{1}{\pi(x-y)}\left(\frac{\partial}{\partial x}-\frac{\partial}{\partial y}\right) M_{n}\left(Z_{n} x\right) M_{n}\left(Z_{n} y\right)\right\} \\
& \quad=\frac{Z_{n}^{2}}{\pi}\left[M_{n}^{\prime}\left(2 Z_{n} r\right) M_{n}^{\prime}\left(2 Z_{n} r\right)-M_{n}\left(2 Z_{n} r\right) M_{n}^{\prime \prime}\left(2 Z_{n} r\right)\right] \equiv Z_{n^{\prime}}^{2} \mathscr{H}_{n}\left(2 Z_{n} r\right),
\end{aligned}
$$

with the analog for the Sturmian functions having $-i k$ in place of $Z_{n}$. Thus,

$$
\begin{aligned}
K(\mathbf{r}, \mathbf{r}, \beta)= & \sum_{n=1}^{\infty} Z_{n}^{3} \mathscr{H}_{n}\left(2 Z_{n} r\right) e^{\beta Z_{n}^{2} / 2}+K_{0}(\mathbf{r}, \mathbf{r}, \beta) \\
& +\frac{i}{2 \pi} \sum_{n=1}^{\infty} \int_{-\infty}^{\infty} \frac{(-i k)^{2} Z_{n} \mathscr{M}_{n}(-2 i k r)}{k-i Z_{n}} e^{-\beta k^{2} / 2} d k
\end{aligned}
$$

The partition function is the integral over volume, which we can take as a sphere of radius $R$ :

$$
q=\int_{0}^{R} K(\mathbf{r}, \mathbf{r}, \beta) 4 \pi r^{2} d r
$$
follows:

We identify the discrete and continuum contributions to the electronic partition function as

$$
q_{\mathrm{elec}}=q_{D}+q_{C} e^{-\beta Z^{2} / 2}
$$

As defined here, the discrete part $q_{D}$ is based on the $n=1$ ground state as the energy zero, while $q_{C}$ takes its energy zero at the onset of the continuum.

If we were to evaluate the integral (29) over all space (with $R=\infty$ ), the normalization of the discrete functions would imply 


\section{$\mathbf{D}_{\mathbf{n}}(\mathbf{r})$}

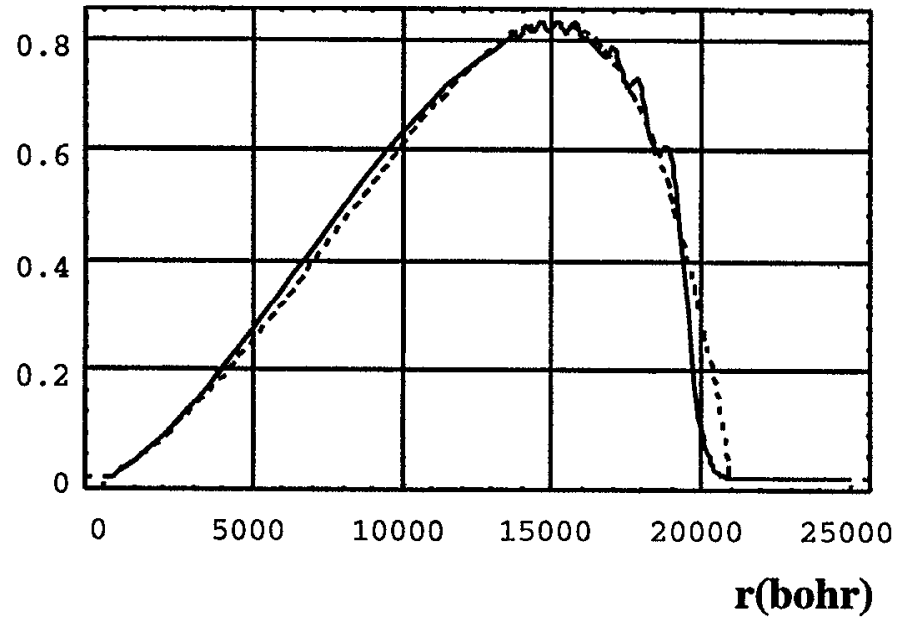

FIG. 1. Solid curve: radial distribution function for $n=100$. Dashed curve: universal reduced form for $D_{n}(r)$ as $n \rightarrow \infty$. See Ref. 15.

$$
\int_{0}^{\infty} Z_{n^{2}}^{3} \mathscr{H}_{n}\left(2 Z_{n} r\right) 4 \pi r^{2} d r \equiv \int_{0}^{\infty} D_{n}(r) d r=n^{2}
$$

consistent with the $n^{2}$ orbital degeneracy of hydrogenic levels. We require instead

$$
q_{D}=\sum_{n=1}^{\infty} e^{\beta\left(Z_{n}^{2}-Z^{2}\right) / 2} \int_{0}^{R} D_{n}(r) d r .
$$

The generalized radial distribution functions $D_{n}(r)$ have been shown ${ }^{15}$ to approach a universal reduced form as $n \rightarrow \infty$, apart from small oscillations, as illustrated in Fig. 1 for the case $n=100$. The integrals can be approximated by

$$
\int_{0}^{R} D_{n}(r) d r \approx n^{2} e^{-\alpha n^{2}}, \quad \alpha=1.23073 / Z R
$$

Thus

$$
\begin{aligned}
q_{D} & \approx 1+\sum_{n=2}^{\infty} n^{2} e^{-\alpha n^{2}} e^{\beta\left(Z_{n}^{2}-Z^{2}\right) / 2} \\
& \approx 1+e^{-\beta Z^{2} / 2}\left[\left(\frac{\sqrt{\pi}}{8 \alpha^{3 / 2}}+\frac{i \sqrt{\pi}}{4 \alpha}\right) e^{-i Z \sqrt{2 \alpha \beta}} \operatorname{erfc}\left(2 \sqrt{\alpha}-\frac{i Z}{2} \sqrt{\frac{\beta}{2}}\right)+\text { c.c. }\right]
\end{aligned}
$$

Next we evaluate the continuum part $q_{C}$. In analogy with (32), the normalization integral over Sturmian functions reads

$$
\int_{0}^{\infty} \mathscr{H}_{n}(-2 i k r) 4 \pi r^{2} d r=n^{2} /(-i k)^{3}
$$


Again, the integral over all space would give a divergent partition function. Even Watson's formula (25) for the free-particle Green's function would lead to

$$
q_{0}=\frac{1}{2} \sum_{n=1}^{\infty} n^{2}=\infty
$$

To take account of finite volume, we introduce a form factor $f(r)$ into the integrals (35) to provide an appropriate cutoff for $r \gtrsim R$. To calibrate this cutoff in the Sturmian functions, we construct a convergent analog of the free-particle summation, as follows:

$$
q_{0}=(2 \pi \beta)^{-3 / 2} V=\frac{1}{2} \sum_{n=1}^{\infty} n^{2} e^{-\gamma n^{2}} \approx \frac{1}{2} \int_{0}^{\infty} n^{2} e^{-\gamma n^{2}} d n
$$

This implies

$$
\gamma=\left(\frac{\sqrt{\pi}}{8 q_{0}}\right)^{2 / 3}=\frac{\pi^{4 / 3} \beta}{2 V^{2 / 3}}
$$

Consistent with (37), the cut-off normalization integrals are given by

$$
\int_{0}^{\infty} \mathscr{C}_{n}(-2 i k r) f(r) 4 \pi r^{2} d r=n^{2} e^{-r n^{2}} /(-i k)^{-3}
$$

To complete the derivation, we do the integral in Eq. (28) using

$$
\int_{-\infty}^{\infty} \frac{e^{-\beta k^{2} / 2}}{k\left(k-i Z_{n}\right)} d k=\frac{\pi}{Z_{n}}\left[e^{\beta Z_{n}^{2} / 2} \operatorname{erfc}\left(\sqrt{\frac{\beta}{2}} Z_{n}\right)-1\right] .
$$

Thus, finally,

$$
q_{C}=q_{0}-\frac{1}{2} \sum_{n=1}^{\infty} n^{2} e^{-\gamma n^{2}}\left[e^{\beta Z_{n}^{2} / 2} \operatorname{erfc}\left(\sqrt{\frac{\beta}{2}} Z_{n}\right)-1\right]
$$

For sufficiently high temperatures,

$$
q_{C} \approx q_{0}\left[1+\frac{(2 \beta)^{1 / 2} Z}{\pi^{5 / 6} q_{0}^{1 / 3}}-\frac{\pi^{1 / 3} \beta Z^{2}}{4 q_{0}^{2 / 3}}\right]
$$

In Fig. 2 we plot the discrete and continuum contributions to the standard-state electronic partition function $q_{\text {elec }}^{\ominus}$ [cf. Eq. (30)] between $10^{3}$ and $10^{8} \mathrm{~K}$. The standard state volume is given by the

$$
V^{\ominus}=N_{A} k T / p^{\ominus}=5.5375 \times 10^{26} T \text { bohr }^{3},
$$

with $p^{\ominus}=1$ bar. For $T \leq 4000 \mathrm{~K}, q \approx 1$, as expected, since for hydrogen $\left(E_{2}-E_{1}\right) / k \approx 1.15 \times 10^{5}$ $\mathrm{K}$. However, the continuum, once it becomes active, totally overwhelms the discrete contribution, a consequence of the immensely larger density of states in the continuum. Moreover, $q_{C}$ is very well approximated at high temperature by $q_{0}$, since the sum in Eq. (41) gocs to zcro as $\beta \rightarrow 0$. 


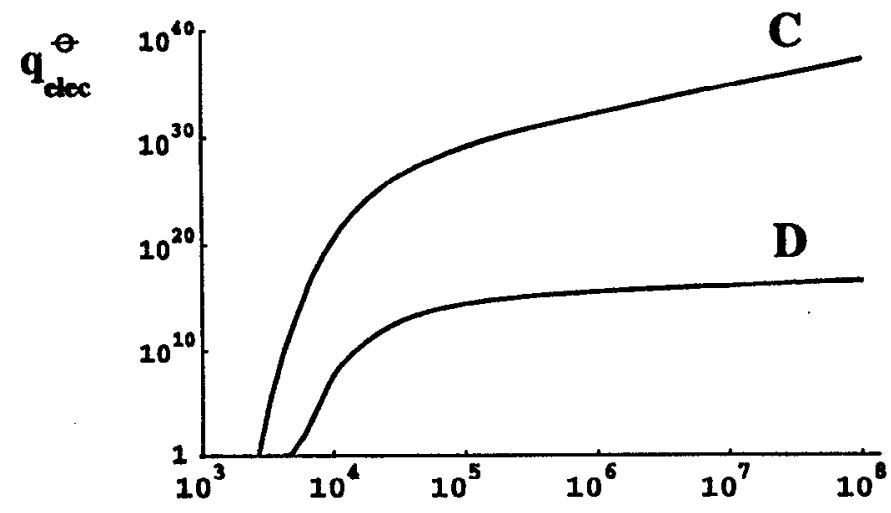

$\mathbf{T}(\mathbf{K})$

FIG. 2. Discrete $(D)$ and continuum $(C)$ contributions to the standard hydrogen partition function: $q_{D}^{\ominus}$ and $q_{C}^{\ominus} e^{-\beta E_{\mathrm{H}} / 2}$, respectively. The discrete contribution is well approximated here by $q_{D}^{\ominus} \approx 1+\left(\sqrt{\pi} / 4 \alpha^{3 / 2}\right) e^{-\beta E_{\mathrm{H}} / 2}$.

\section{SAHA-BOLTZMANN EQUATION}

Throughout this paper we have assumed perfect-gas behavior of hydrogen atoms, protons, and electrons. Consistent with this picture, electronic wave functions of high quantum number should penetrate neighboring atoms with no ill effect. In the atmospheres of typical stars, the particle density is sufficiently low so that the perfect-gas equation of state is approximately valid-at least for neutral atoms. The actual physical situation for charged particles is more complicated, involving Debye screening, radiation, hydrodynamic effects, and possibly deviations from thermodynamic equilibrium.

It is not our purpose to treat astrophysical or other applications in any realistic detail. However, to give our results a more concrete perspective, we refer to the equilibrium between bound and ionized hydrogen atoms, in a hypothetical perfect-gas stellar atmosphere:

$$
\mathrm{H} \rightleftharpoons \mathrm{H}^{+}+e .
$$

By the Saha-Boltzmann equation: ${ }^{16}$

$$
K_{p}=\frac{\left(p_{\mathrm{H}^{+}} / p^{\ominus}\right)\left(p_{e} / p^{\ominus}\right)}{\left(p_{\mathrm{H}} / p^{\ominus}\right)}=\frac{\left[q^{\ominus}\left(\mathrm{H}^{+}\right) / N_{A}\right]\left[q^{\ominus}(e) / N_{A}\right]}{\left[q^{\ominus}(\mathrm{H}) / N_{A}\right]} e^{-\beta E_{\mathrm{H}} / 2} .
$$

We can identify $q(\mathrm{H})$ with $q_{\text {trans }}(\mathrm{H}) \times q_{D}$ and $q(e)$ with $q_{C}$. The hydrogen ion factor $q\left(\mathrm{H}^{+}\right)$is just a free-particle partition function, which effectively cancels the translational factor in $q(\mathrm{H})$. The electron spin degeneracy (factor 2 ) in $q(\mathrm{H})$ and $q(e)$ also cancels. Assuming local thermodynamic equilibrium and perfect-gas behavior, the Saha-Boltzmann equation for hydrogen thus reduces to

$$
K_{p}=\frac{q_{C}^{\ominus} / N_{A}}{q_{D}^{\ominus}} e^{-\beta E_{\mathrm{H}} / 2}
$$

Putting in the component partition functions as computed in Fig. 2, we plot the equilibrium constant as a function of temperature in Fig. 3. We note that in the Sun, only about $10 \%$ of the electron density comes from hydrogen atoms, the remainder being contributed by ionization of heavier elements. Such details would have to be taken in account in astrophysical applications of the Saha-Boltzmann theory, which we leave to other workers. 


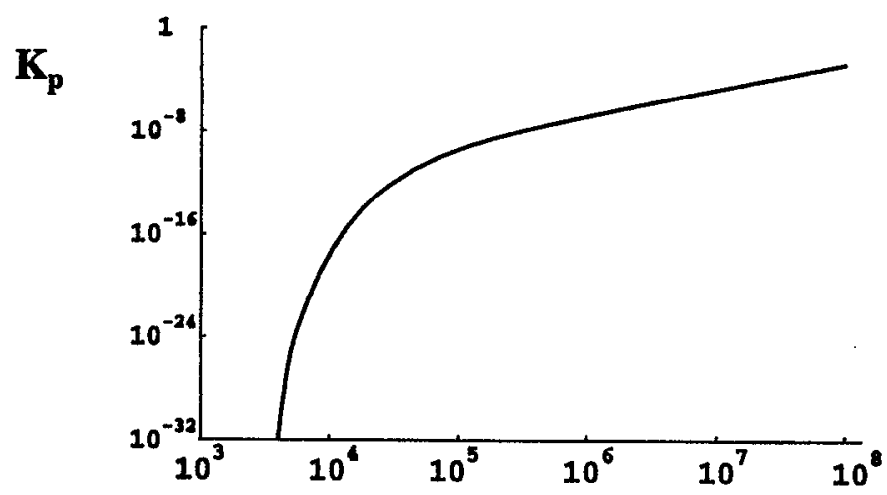

$\mathbf{T}(\mathbf{K})$

FIG. 3. Saha-Boltzmann equilibrium constant for ionization of atomic hydrogen.

\section{ACKNOWLEDGMENT}

I would like to thank Professor Lawrence L. Lohr for several stimulating discussions on the hydrogen partition function.

${ }^{1}$ K. Herzfeld, Ann. Phys. 51, 251 (1916); H. C. Urey, Astrophys. J. 49, 1 (1924); E. Fermi, Z. Phys. 26, 54 (1924); M. Planck, Ann. Phys. 75, 673 (1924); R. H. Fowler, Philos. Mag. 1, 845 (1926); R. H. Fowler, Statistical Mechanics (Cambridge University, Cambridge, 1936), p. 572ff; E. P. Wigner, Phys. Rev. 94, 77 (1954); R. E. Treves, ibid. 102, 1533 (1956); B. F. Gray, J. Chem. Phys. 36, 1801 (1962); ibid 55, 2848 (1971); M. McChesney, Can. J. Phys. 42, 2473 (1964); S. J. Strickler, J. Chem. Ed. 43, 364 (1966); C. A. Rouse, Phys. Rev. 163, 62 (1967); A. Wehrl, Rev. Mod. Phys. 50, 221 (1978); M. Grabowski, Rep. Math. Phys. 23, 19 (1986).

2 J. W. Gibbs, Elementary Principles in Statistical Mechanics (Yale University, New Haven, 1902; reprinted by Dover, New York, 1960), p. 35.

${ }^{3}$ See, for example, R. P. Feynman and A. R. Hibbs, Quantum Mechanics and Path Integrals (McGraw-Hill, New York, 1965).

${ }^{4}$ S. M. Blinder, Phys. Rev. A 43, 13 (1991).

${ }^{5}$ A. I. Larkin, Zh. Exper. Teoret. Fiz. 38, 1896 (1960); English translation: Sov. Phys-JETP 11, 1363 (1960); W. Ebeling and R. Sändig, Ann. Phys. 28, 289 (1973).

${ }^{6}$ C. A. Rouse, Astrophys. J. 272, 377 (1983).

${ }^{7}$ S. M. Blinder, Phys. Rev. $\Lambda$ 37, 973 (1988).

${ }^{8}$ L. Hostler and R. H. Pratt, Phys. Rev. Lett. 10, 469 (1963); L. Hostler, J. Math. Phys. 5, 591 (1964).

${ }^{9}$ S. M. Blinder, Int. J. Quantum Chem. S 18, 293 (1984), and references therein.

${ }^{10} \mathrm{H}$. Buchholz, The Confluent Hypergeometric Function (Springer, New York, 1969).

${ }^{11}$ S. M. Blinder, International Reviews of Science, Vol I, Theoretical Chemistry (Butterworths, London, 1975).

${ }^{12}$ E. T. Whittaker and G. N. Watson, A Course in Modern Analysis (Cambridge University, Cambridge, 1958), pp. $134-136$

${ }_{13}^{13}$ L. Hostler, J. Math. Phys. 11, 2966 (1970).

${ }^{14}$ H. Buchholz, Ref. 10 , p. 140 .

${ }^{15} \mathrm{~S}$. M. Blinder, J. Math. Chem. 14, 319 (1993).

${ }^{16}$ See, for example, K. R. Lang, Astrophysical Formulae (Springer, New York, 1974), pp. $244 \mathrm{ff}$. 